\title{
Real-Time Adherence Monitoring for HIV Antiretroviral Therapy
}

\author{
Jessica E. Haberer • Josh Kahane • Isaac Kigozi • \\ Nneka Emenyonu · Peter Hunt · Jeffrey Martin • \\ David R. Bangsberg
}

Published online: 31 August 2010

(C) The Author(s) 2010. This article is published with open access at Springerlink.com

\begin{abstract}
Current adherence assessments typically detect missed doses long after they occur. Real-time, wireless monitoring strategies for antiretroviral therapy may provide novel opportunities to proactively prevent virologic rebound and treatment failure. Wisepill, a wireless pill container that transmits a cellular signal when opened, was pilot tested in ten Ugandan individuals for 6 months. Adherence levels measured by Wisepill, unannounced pill counts, and selfreport were compared with each other, prior standard electronic monitoring, and HIV RNA. Wisepill data was initially limited by battery life and signal transmission interruptions. Following device improvements, continuous data was achieved with median (interquartile range) adherence levels of $93 \%(87-97 \%)$ by Wisepill, $100 \%(99-100 \%)$ by unannounced pill count, $100 \%(100-100 \%)$ by self-report, and
\end{abstract}

J. E. Haberer · N. Emenyonu · D. R. Bangsberg

Massachusetts General Hospital Center for Global Health,

Boston, MA, USA

J. E. Haberer $(\bowtie) \cdot$ J. Kahane $\cdot$ N. Emenyonu •

D. R. Bangsberg

Harvard Initiative for Global Health, 104 Mt. Auburn Street,

3rd floor, Cambridge, MA 02138, USA

e-mail: jhaberer@partners.org

J. E. Haberer · D. R. Bangsberg

Ragon Institute of Massachusetts General Hospital,

Massachusetts Institute of Technology and Harvard University,

Charlestown, MA, USA

I. Kigozi · N. Emenyonu · D. R. Bangsberg

Mbarara University of Science and Technology,

Mbarara, Uganda

P. Hunt $\cdot$ J. Martin

University of California, San Francisco, San Francisco,

CA, USA
92\% (79-98\%) by prior standard electronic monitoring. Four individuals developed transient, low-level viremia. After overcoming technical challenges, real-time adherence monitoring is feasible for resource-limited settings and may detect suboptimal adherence prior to viral rebound.

Keywords Real-time adherence monitoring . Wireless technology $\cdot$ Antiretroviral therapy

\section{Introduction}

Current approaches to antiretroviral therapy (ART) adherence monitoring include various forms of structured patient interview (also known as self-report), pill counts, pharmacy refill, and electronic monitoring [1]. In practice, all of these methods are assessed on an intermittent basis, such that missed doses are detected several weeks to months after they occur. They are therefore often unable to direct adherence interventions before resumption of viral replication, which can lead to treatment failure and drug resistance. Loss of viral suppression may begin as early as $48 \mathrm{~h}$ after a lapse in adherence, and a 15-day interruption confers a $50 \%$ chance of virologic failure among suppressed individuals on non-nucleoside reverse transcriptase inhibitor therapy [2]. Real-time adherence monitoring could allow for the detection of adherence lapses and interventions to resume treatment prior to the development of virologic rebound and drug resistance, which has not previously been possible [3]. This real-time approach is particularly important in settings with limited treatment options in that resources for behavioral and structural interventions could be directed specifically at identified adherence lapses.

The Wisepill adherence monitor (see Fig. 1; Wisepill Technologies, Cape Town, South Africa) communicates 


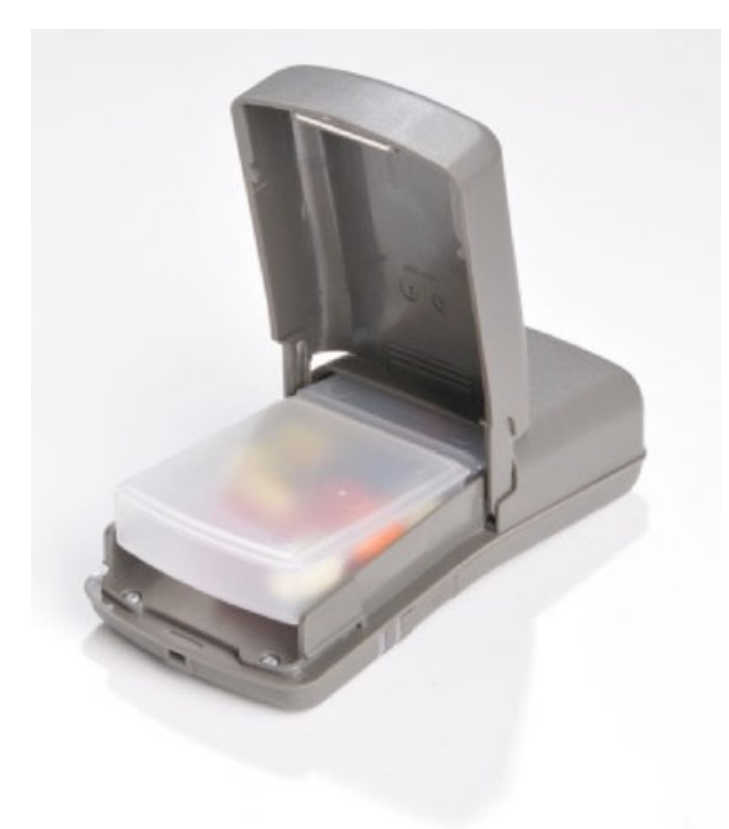

Fig. 1 The Wisepill device

dosing behavior in real-time by transmission of a patient identifier and date-time stamp over existing cellular networks when the container is opened to take medications. Similar real-time monitoring devices are being pilot tested for various healthcare uses, such as infection control and oral hygiene, in developed settings [4, 5]. Because cellular network coverage is becoming ubiquitous globally [6], including large segments of Africa, the technical infrastructure now exists for real-time adherence monitoring in resource-limited settings. While excitement abounds for wireless technologies to support healthcare delivery, especially in developing settings, few studies have provided evidence of feasibility, acceptability, and performance [7].

This study presents proof-of-concept data from the first 6 months of wireless ART adherence monitoring among ten patients in rural Uganda.

\section{Methods}

\section{Study Site}

This pilot study took place in Mbarara, Uganda, which is a rural area located in southwestern part of the country near the borders with Rwanda and the Democratic Republic of the Congo.

\section{Study Population}

The ten participants were recruited from the Ugandan ART Rural Treatment Outcomes (UARTO) Study, an existing prospective structured interval cohort of 500 adults followed for ART adherence at the Mbarara University of Science and Technology (MUST). Participants had prior adherence monitoring with monthly visual analog scale (VAS) for doses taken over the previous 30 days, selfreported recall of doses missed over the previous 3 days, and unannounced home-based pill count, as well as electronic monitoring with the medication event monitoring system (MEMS) for at least 1 year. Adequate cellular signal (at least three of five bars) was confirmed at all participants' homes prior to enrollment in the study.

\section{Wireless Adherence Monitoring Device and System Specifications}

Wisepill holds approximately 30 large pills or 60 small pills in a two-compartment inner container and is powered by a $1,100 \mathrm{~mA}$ lithium polymer rechargeable battery (Great Power Battery Ltd, Hong Kong). Every time the device is opened, a cellular signal is sent and recorded in real-time on a web-based server, which is housed in Cape Town, South Africa. The data is then immediately accessible to research staff via a secure Internet interface. Power failure is mitigated with a signaling subsystem in which non-volatile EEprom (flash memory) maintains data for later transmission if connectivity is lost. Each Wisepill device contains a SIM card, and data are transmitted primarily by general packet radio service (GPRS) to a web-based server. Data transfer may also occur via short message service (SMS); however, GPRS is preferred because it is less expensive and, unlike SMS, does not delete data until receipt is confirmed by the server. A forwarder (Wisepill Technologies) is required to transfer the SMS signal to the server and is located physically in Mbarara, Uganda. In addition to recording device openings, the Wisepill signal reports the remaining battery power for the device, airtime on the SIM card, and strength of the signal.

Improvements were made to the Wisepill devices in between the two 3-month study periods. Specifically, the hardware for the forwarder was upgraded and monitoring of the forwarder function was enabled through the server. The Wisepill battery life was also expanded from approximately 6 weeks to 4 months (assuming two device openings per day) by maximizing the efficiency of sleep modes in between events.

\section{Signal Lapses}

Lapses in Wisepill signals lasting greater than $48 \mathrm{~h}$ were investigated by phone and/or home visit to determine if the cause was a technical failure, behavioral, or unclear. Technical failure was classified as low battery power, low airtime on the device SIM card, forwarder malfunction, 
device malfunction, or other. Behavioral causes were classified based on patient interview as missed doses (e.g. forgetting, lack of drug supply), intentional non-use (e.g. storage of pills in an alternate container), or other. The cause of the interruption was classified as unclear if no technical failure could be identified and the participant reported opening the device to take pills.

\section{Adherence Measures}

Adherence data was collected for all participants over two 3-month periods between March and December 2009. We calculated an adjusted Wisepill adherence to account for technical failures of the device or loss of signal. The time period during the technical lapse was censored, whereas lapses in the absence of a clear technical failure were classified as missed doses. Adherence was also measured monthly with a 30-day VAS, a 3-day self-reported recall of missed doses, and an unannounced pill count conducted in the participants' homes. Pill count adherence levels were truncated at $100 \%$, as adherence over this amount typically reflects lost or shared medication and this method of adherence measurement is inherently unable to account for such phenomena. MEMS adherence data was available for the 3 months just prior to Wisepill implementation in six participants. MEMS data was not available in the other four participants due to device malfunction or loss.

\section{Acceptability}

Acceptability of Wisepill was determined 1 month after initiation of the pilot through the use of a quantitative and open-ended questionnaire. Questions addressed the participants' opinions of using the device, including ease or difficulty of use; how they store the device and use it during travel; potential stigma and loss of confidentiality from using the device; appropriateness of this device in a poor rural setting; and opinions of real-time monitoring ultimately to be coupled with real-time intervention. All questions were translated into the local language of Runyankole and back translated to English for accuracy.

\section{HIV RNA Levels}

HIV RNA was determined a median of 17 days (IQR 6-36) days prior to baseline and approximately every 3 months thereafter using the Roche Amplicor Assay with a lower level of detection of 40 copies/ml [8]. HIV RNAs were missing for two participants at the end of the study period.
Ethical Review

This study was approved by the ethical review boards at MUST, the Partners Human Research Committee at Massachusetts General Hospital, and the Uganda National Council for Science and Technology.

\section{Results}

Participants

The median age for the ten participants at enrollment was 38 (interquartile range 34-46) years, eight were female, four were employed away from home, two lived more than $20 \mathrm{~km}$ from the clinic, nine took ART twice daily, and one took ART once daily. Eight participants took a three-drug fixed dose combination pill (seven took zidovudine/lamivudine/ nevirapine; one took stavudine/lamivudine/nevirapine). The remaining two took a two-drug fixed dose combination pill (zidovudine/lamivudine) plus efavirenz, storing one pill in the Wisepill and the other pill in a standard medication bottle. The mean time on ART was 19.2 months at enrollment into the pilot. All participants had undetectable HIV RNA less than 40 copies $/ \mathrm{ml}$ at enrollment, except one who had 60 copies $/ \mathrm{ml}$.

\section{Wisepill Data Transmission and Adherence Lapses}

Approximately $90 \%$ of Wisepill transmissions occurred via GPRS. Adequate cellular signal was reported for all devices except one, which had intermittent gaps. During the first 3 months of the study, a total of 1,959 events were expected for all ten participants. Technical problems consisted of 23 battery failures and seven forwarder malfunctions, causing a loss of 192 events (9.8\%; Table 1). Twenty of the battery failures and three of the forwarder malfunctions resulted in lapses in the Wisepill signal of greater than $48 \mathrm{~h}$. An additional three lapses occurred for unclear reasons. The median duration of these lapses was 3.1 days (IQR 2.3-3.6).

During the second 3 months of the study, the only technical problem was a single battery failure after

Table 1 Technical problems detected in the first and second threemonth periods of the study

\begin{tabular}{lll}
\hline Technical problems & First & Second \\
& 3 months & 3 months \\
\hline Battery failures & 23 & 1 \\
Forwarder malfunctions & 7 & 0 \\
Total & 30 & 1 \\
Percentage of expected events & 9.8 & 0.3 \\
\hline
\end{tabular}


2.5 months of use. There were no problems with the forwarder. Four lapses in the Wisepill signal of greater than $48 \mathrm{~h}$ occurred. One lapse was due to the battery failure. Another was intentional because the participant did not want to travel with the Wisepill device and put 1-week's worth of pills in his pocket. The causes of the other two lapses are unclear. The median duration of the 48-h lapses was 3.7 days (IQR 2.7-5.1).

One week following the conclusion of the pilot, all devices ceased to work on the same day. Thorough investigations revealed that the SIM cards in the Wisepill device had expired because no airtime had been added in the previous 90 days.

\section{Adherence Levels}

Median adherence levels by each measure are shown in Fig. 2. Eight of 54 (14.8\%) unannounced pill counts were truncated to $100 \%$ with a maximum recorded value of $104.3 \%$.

\section{HIV RNA Suppression}

The participant with a baseline HIV RNA of 60 copies/ml had two subsequently measurements at 51 copies $/ \mathrm{ml}$ and undetectable. Three additional patients had transient viral rebounds $(58,134$, and 627 copes $/ \mathrm{ml})$ in the second 3 -month period, which subsequently became undetectable. Figure 3 shows the adherence dot plots in the 14 days prior to each of these detectable HIV RNA measurements. No Wisepill interruptions of greater than $48 \mathrm{~h}$ occurred, although between five and eight missed events without a 48-h interruption were observed in three of the four participants. When assessing the nine participants during the first 3-month period and the seven participants during the second 3-month period who had undetectable HIV RNA, one participant had one Wisepill interruption that was attributed to a battery failure and $13(81 \%)$ participants had between one and nine missed events without a 48-h interruption during the 14 days prior to HIV RNA testing.

\section{Acceptability}

All participants described Wisepill as "easy or very easy" or "convenient or very convenient" to use. They all felt the device attracted attention, but the attention did not bother them primarily because the device did not look like a traditional pill container. Wisepill did not lead to any reported disclosures of HIV status. When asked "How do you feel about someone monitoring how you are taking your pills every day?" all participants responded "ok" or "liked it". One participant felt it improved adherence. Concerns about the device included a limited pill capacity and the need to change the battery. One participant with impaired vision had trouble interpreting the meaning of the flashing lights during signal transmission, whereas others found the lights reassuring. Three participants expressed concern about traveling with Wisepill primarily because they were concerned about losing or damaging such an expensive device. Such concern resulted in one participant not using the device for 1 week, as noted above.
Fig. 2 Median adherence levels by multiple measures averaged over the two 3-month study periods. * MEMS data was recorded during the 3 months just prior to the initiation of this study

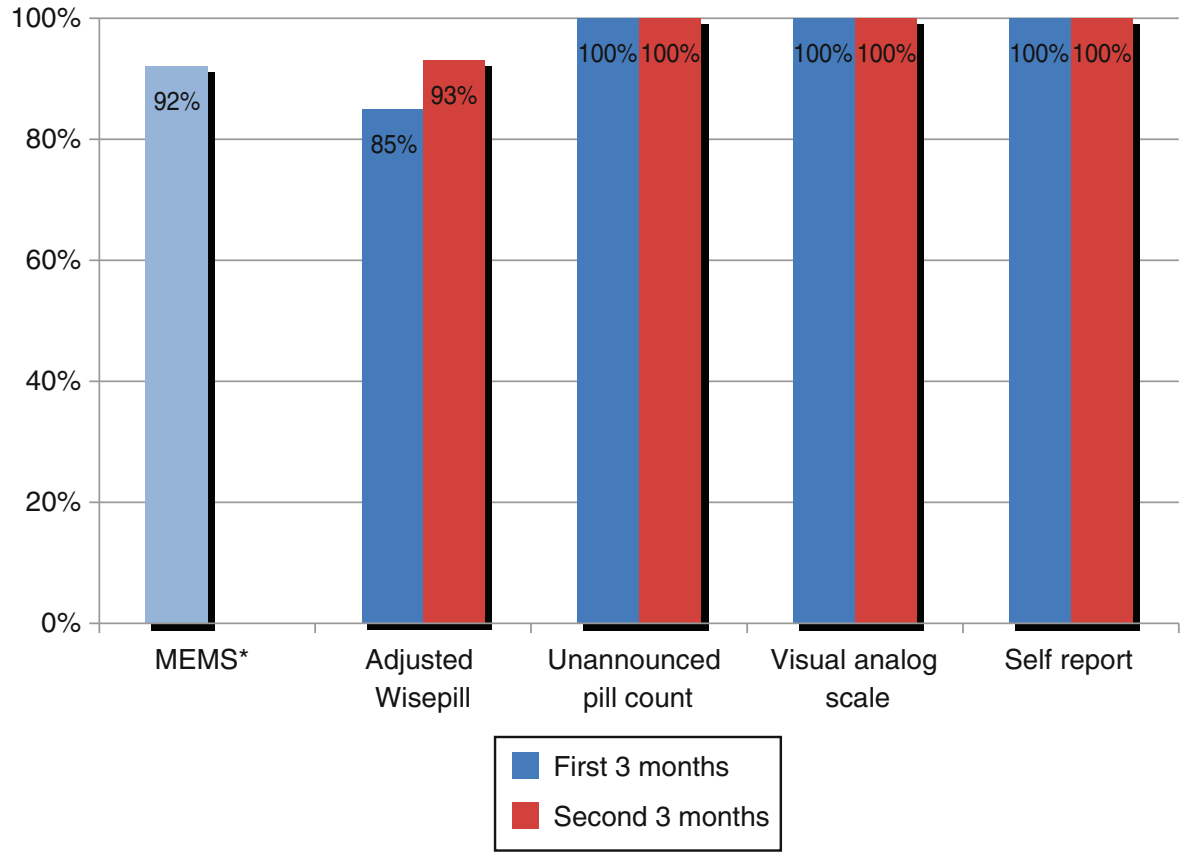



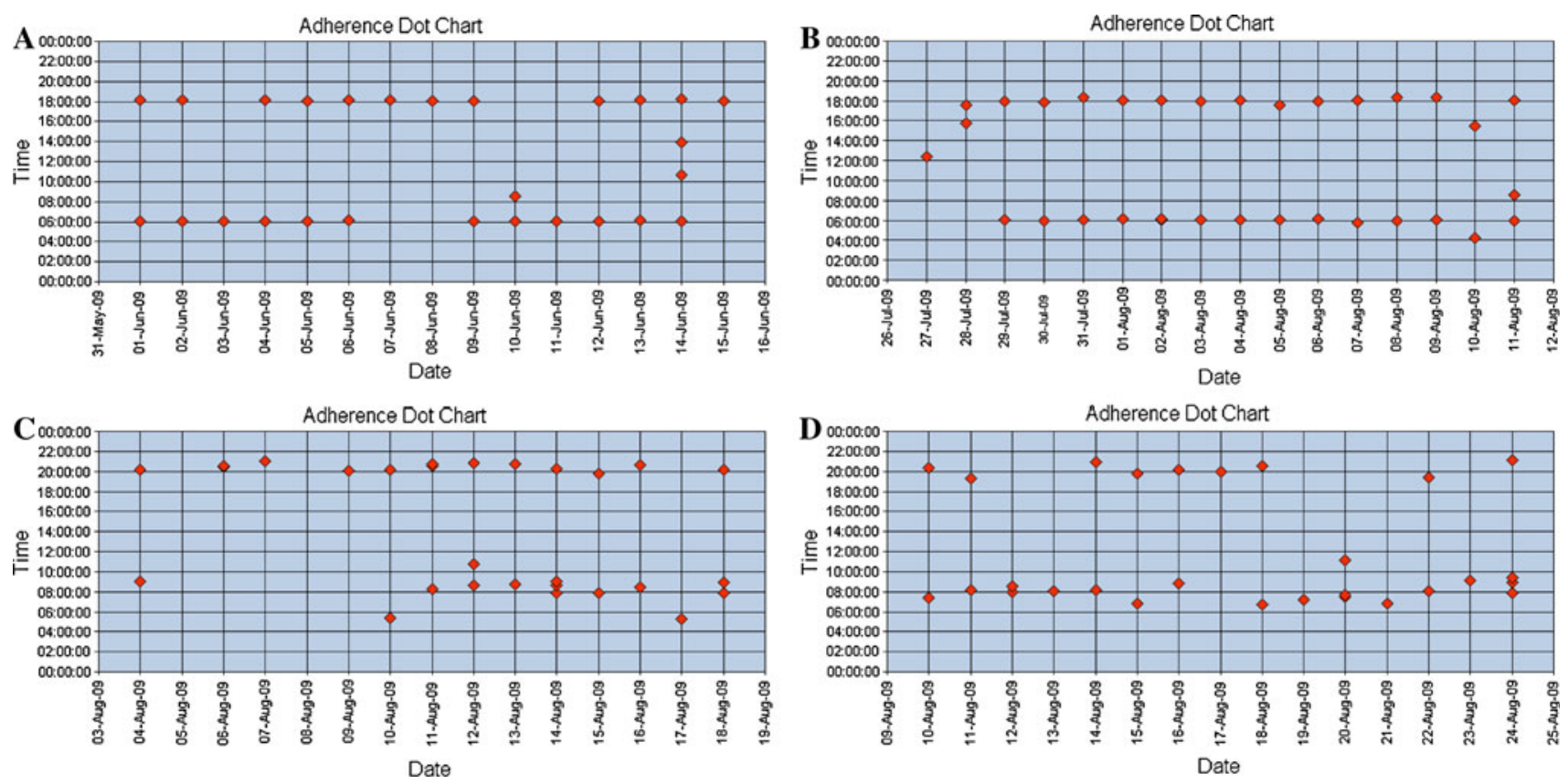

Fig. 3 Adherence dot plots for the 14 days prior to detection of the transient, low-level viremia seen in four participants. a HIV RNA 627 copies/ml (6/15/09; first 3-month period); b HIV RNA 134 copies/ml

(8/11/09; second 3-month period); c HIV RNA 58 copies/ml (8/18/09; second 3-month period); d HIV RNA 51 copies/ml (8/24/09; second 3-month period)

\section{Discussion}

The results of this pilot study suggest that real-time adherence monitoring is feasible in a resource-limited setting. The initial 3 months of data collection revealed significant technical problems; however, most were resolved during the course of the study. Minor modifications to the sleep mode extended the battery life to nearly 4 months for almost all issued devices, and SMS signal transmission became reliable through upgrades to the forwarder. The SIM card expiration after the conclusion of this pilot study occurred despite monitoring the level of airtime available. Research staff were unaware that the network coverage provider automatically inactivates the SIM cards if no airtime is added during a 6-month period, and contract prepayment plans are not available. This finding underscores the importance of in field-testing for novel technologies.

Although the adherence levels assessed with Wisepill were similar to those made with prior electronic monitoring through MEMS, they were lower than the adherence levels determined by unannounced pill counts and self-report. It is not known which measure is correct, as there is no gold standard for adherence measurement. Moreover, the sample size in this pilot study is too small to determine the statistical significance of any differences among the measurements. The apparent discrepancy between unannounced pill count and electronic monitoring, both of which are objective measures, may be due to patients

returning unused medications to the pharmacist during refills and other inconsistencies during medication disbursement. Alternatively, participants may have removed more than one pill at a time or further technical problems with Wisepill could have gone undetected, thus leading to falsely low adherence measurements. Undetected technical problems may also explain some of the difference in Wisepill adherence in the first and second 3-month periods. Additionally, the adherence levels as measured by both MEMS and Wisepill were lower than similar data from other studies $[9,10]$, which may reflect the relatively long duration of treatment of the participants in this study. The decline in adherence is consistent with data showing that highly adherent individuals may experience a modest decline in adherence over time $[9,11]$.

Both 3-month time periods had similar rates of lapses in the Wisepill signal that could not be classified as a specific technical or behavioral cause. These unclear lapses may reflect missed doses that were not reported due to social desirability bias against disclosure of missed doses. This type of bias is common and can lead to under-reporting of incomplete adherence [12]. Interventions to address the lapses detected through Wisepill will therefore need to consider culturally acceptable means of determining both the cause of missed doses and appropriate responses. It is also possible that lapses of unclear cause could indicate undetected technical failures, despite the ongoing checks of signal strength, airtime, and battery power, as well as 
transmission of signals stored during temporary loss of the cellular network. To further decrease the likelihood of technical failures, the manufacturer is currently adding an automated daily signal, which will serve as a positive control of the device's functionality.

Interventions targeted to individuals with known adherence lapses have been shown to be more effective than untargeted interventions [13, 14], and real-time adherence monitoring could be used to direct interventions to specific missed doses prior to virologic rebound. Such a "just-in-time" approach may increase effectiveness further $[15,16]$. For example, automated SMS reminders could be triggered by missed or late doses, or a community health worker could call or personally visit a patient during an adherence lapse to intervene before viral rebound.

The pilot data showed that real-time adherence monitoring was acceptable to all participants. Many found Wisepill desirable, easy to use, and/or thought it helped them remember to take their doses. One limitation to these findings is that the study population consisted of individuals already accustomed to the use of MEMS caps, which may select for acceptance of the Wisepill device as well. The generalizability and sustainability of this initial enthusiasm will have to be determined in larger populations over time, and these assessments will be critical for the scalability of this technology. Several theories and models of technology acceptance have been developed to best understand the experience of the end user, such as the Technology Acceptance Model and the Unified Theory of Acceptance and Use of Technology [17-19]. These models build upon the theory of reasoned action [20] and include elements such as perceived usefulness, perceived ease of use, output quality, social influence, attitude, behavioral intention to use, and actual use. Moreover, standard electronic medication containers have known facilitators and barriers to uptake, such as the container acting as a reminder and incompatibility with pill boxes, respectively [21]. While this study was too small in scope to inform a theoretical model, future efforts to implement real-time adherence monitoring should consider underlying theory and previous research.

Several individuals in this study experienced transient low-level viremia, consistent with blips [22]. While transient low-level viremia has been associated with transient periods of incomplete adherence [23], we were unable to assess such an association in our small pilot study. This study does, however, provide proof-of-concept that participants can be contacted during potential adherence lapses. In future studies, HIV RNA could be assessed during the adherence lapse to determine how quickly viral rebound occurs in this setting and to what extent it can be re-suppressed without the development of drug resistance.
Studies have shown that ART adherence predicts viral rebound, drug resistance, and morbidity and mortality from HIV/AIDS [24-26]. Furthermore, relatively crude pharmacy refill adherence monitoring may be a better predictor of viral rebound than the standard practice of CD4 response for detecting treatment failure [27]. More accurate adherence monitoring, such as can be achieved through a realtime approach, may help prioritize limited resources for HIV RNA monitoring to those at increased risk for viral bound.

The findings of this study reflect a small sample in a research context. Future research should aim to expand the number of participants and establish the data and management structure necessary to test the efficacy of detecting virologic rebound in real time, and ultimately how such real-time detection of virologic rebound can be linked to interventions that improve patient outcomes in clinical practice. Cost is another concern. Although the Wisepill device currently costs US $\$ 185$; mass production could lower the price to US\$20-30. Significant cost savings could be achieved through economies of scale, as well as targeted use of HIV RNA monitoring and reduced need for second line therapies. Such savings could bring real-time adherence monitoring into the same cost range as CD4 monitoring.

In sum, real-time adherence monitoring defines a feasible and acceptable strategy to proactively prevent rather than reactively respond to virologic treatment failure and thereby extend the duration of first-line therapy. Further studies should build on this pilot data to determine the efficacy of real-time intervention for sustained adherence and its potential to act as a surrogate for HIV RNA testing.

Acknowledgments This study was made possible by the generous contributions of Mark and Lisa Schwartz, in addition to RO-1, NIMH 54907. Jessica Haberer was supported by NIMH K-23 087228, and David Bangsberg was supported by NIMH K-24 087227. The authors would like to thank the study participants; Lloyd Marshall and Paul Bailey at Wisepill; and research assistants Abdon Birungi, Emmanuel Byaruhanga, Joy Kabasindi, Esther Susan Kanyunyuzi, Chris Mulokozi, and Victoria Nafuka. The manufactures of Wisepill had no role in the analysis or preparation of the manuscript and the authors have no financial interest in Wisepill.

Open Access This article is distributed under the terms of the Creative Commons Attribution Noncommercial License which permits any noncommercial use, distribution, and reproduction in any medium, provided the original author(s) and source are credited.

\section{References}

1. Kerr T, Walsh J, Lloyd-Smith E, et al. Measuring adherence to highly active antiretroviral therapy: implications for research and practice. Curr HIV/AIDS Rep. 2005;2(4):200-5.

2. Parienti JJ, Das-Douglas M, Massari V, et al. Not all missed doses are the same: sustained NNRTI treatment interruptions 
predict HIV rebound at low-to-moderate adherence levels. PLoS One. 2008;3(7):e2783.

3. Bangsberg DR, Deeks SG. Spending more to save more: interventions to promote adherence. Ann Intern Med. 2010;152(1): 54-6.

4. Boyce JM, Cooper T, Dolan MJ. Evaluation of an electronic device for real-time measurement of alcohol-based hand rub use. Infect Control Hosp Epidemiol. 2009;30(11):1090-5.

5. Walji MF, Coker O, Valenza JA, et al. A persuasive toothbrush to enhance oral hygiene adherence. AMIA Annu Symp Proc. 2008; 1167.

6. United Nations International Telecommunication Union. Measuring the information society - the ICT development index 2009. http://www.itu.int/ITU-D/ict/publications/idi/2009/material/IDI 2009_w5.pdf. Accessed 10 May 2010.

7. Kahn JG, Yang JS, Kahn JS. "Mobile" health needs and opportunities in developing countries. Health Aff. 2010;29(2): 252-8.

8. Schumacher W, Frick E, Kauselmann M, et al. Fully automated quantification of human immunodeficiency virus (HIV) type 1 RNA in human plasma by the COBAS AmpliPrep/COBAS TaqMan system. J Clin Virol. 2007;38(4):304-12.

9. Oyugi JH, Byakika-Tusiime J, Ragland K, et al. Treatment interruptions predict resistance in HIV-positive individuals purchasing fixed-dose combination antiretroviral therapy in Kampala, Uganda. AIDS. 2007;21(8):965-71.

10. Oyugi JH, Byakika-Tusiime J, Charlebois ED, et al. Multiple validated measures of adherence indicate high levels of adherence to generic HIV antiretroviral therapy in a resource-limited setting. J Acquir Immune Defic Syndr. 2004;36(5):1100-2.

11. Byakika-Tusiime J, Crane J, Oyugi JH, et al. Longitudinal antiretroviral adherence in HIV + Ugandan parents and their children initiating HAART in the MTCT-Plus family treatment model: role of depression in declining adherence over time. AIDS Behav. 2009;13(Suppl 1):82-91.

12. Simoni JM, Kurth AE, Pearson CR, et al. Self-report measures of antiretroviral therapy adherence: a review with recommendations for HIV research and clinical management. AIDS Behav. 2006;10(3):227-45.

13. Hart JE, Jeon CY, Ivers LC, et al. Effect of directly observed therapy for highly active antiretroviral therapy on virologic, immunologic, and adherence outcomes: a meta-analysis and systematic review. J Acquir Immune Defic Syndr. 2010;54(2): $167-79$.
14. Amico KR, Harman JJ, Johnson BT, et al. Efficacy of antiretroviral therapy adherence interventions: a research synthesis of trials, 1996 to 2004. J Acquir Immune Defic Syndr. 2006;41(3):285-97.

15. Murtaugh CM, Pezzin LE, McDonald MV, et al. Just-in-time evidence-based e-mail "reminders" in home health care: impact on nurse practices. Health Serv Res. 2005;40(3):849-64.

16. Intille SS. Ubiquitous computing technology for just-in-time motivation of behavior change. Stud Health Technol Inform. 2004;107(Pt 2):1434-7.

17. Holden RJ, Karsh BT. The technology acceptance model: its past and its future in health care. J Biomed Inform. 2010;43(1): 159-72.

18. Davis F. Perceived usefulness, perceived ease of use, and user acceptance of information technology. MIS Q. 1989;13:319-39.

19. Venkatesh V, Morris MG, David GB, et al. User acceptance of information technology: toward a unified view. MIS Q. 2003;27: 425-78.

20. Fishbein M, Ajzen I. Belief, attitude, intention, and behavior: an introduction to theory and research. Reading, MA: AddisonWesley; 1975.

21. Wendel CS, Mohler MJ, Kroesen K, et al. Barriers to use of electronic adherence monitoring in an HIV clinic. Ann Pharmacother. 2001;35(9):1010-5.

22. Havlir DV, Bassett R, Levitan D, et al. Prevalence and predictive value of intermittent viremia with combination HIV therapy. JAMA. 2001;286(2):171-9.

23. Podsadecki TJ, Vrijens BC, Tousset EP, et al. Decreased adherence to antiretroviral therapy observed prior to transient human immunodeficiency virus type 1 viremia. J Infect Dis. 2007; 196(12):1773-8.

24. Bangsberg DR, Hecht FM, Charlesbois ED, et al. Adherence to protease inhibitors, HIV-1 viral load, and development of drug resistance in an indigent population. AIDS. 2000;14(4):357-66.

25. Bangsberg DR, Perry S, Charlesbois ED, et al. Non-adherence to highly active antiretroviral therapy predicts progression to AIDS. AIDS. 2001;15(9):1181-3.

26. Hogg RS, Heath K, Bangsberg D, et al. Intermittent use of triplecombination therapy is predictive of mortality at baseline and after 1 year of follow-up. AIDS. 2002;16(7):1051-8.

27. Bisson GP, Gross R, Bellamy S, et al. Pharmacy refill adherence compared with CD4 count changes for monitoring HIV-infected adults on antiretroviral therapy. PLoS Med. 2008;5(5):e109. 